Discussion There are many ways to speed up systematic review, some at risk of introducing bias. Without clear understanding of the reasons users request rapid review and their expectations for the evidence product, simply speeding the time frame may not address all of user needs for evidence they can implement quickly.

Implications for Guideline Developers/Users Until there is consensus on what the label rapid review describes, users will need to identify their own minimal standards and evaluate adherence. Developers should be clear about user expectations for using the evidence.

\section{RAPID GUIDELINES: A SYSTEMATIC REVIEW}

${ }^{1,2,3} \mathrm{M}$ Falavigna, ${ }^{4}$ I Ikobaltzeta, ${ }^{3} \mathrm{~F}$ Sakhia, ${ }^{3} \mathrm{Y}$ Zhang, ${ }^{3} \mathrm{~N}$ Santesso, ${ }^{5} \mathrm{~S}$ Norris, ${ }^{3,6} \mathrm{H}$ Schünemann. 'Universidade Federal do Rio Grande do Sul, Porto Alegre, Brazil; ${ }^{2}$ IATS - National Institute of Science and Technology for HTA, Porto Alegre, Brazil; ${ }^{3}$ Department of Clinical Epidemiology and Biostatistics. McMaster University, Hamilton, Canada; ${ }^{4}$ OSTEBA - Basque Office for Health Technology Assessment, Vitoria-Gasteiz, Spain; ${ }^{5}$ World Health Organization, Geneve, Switzerland Department of Medicine; ${ }^{6}$ McMaster University Hamilton, Canada

\section{0:1136/bmjgs-2013-002293.63}

Background Guidelines often take two or more years to be developed. This timeframe is not practical for providing guidance in situations when rapid advice is needed.

Objectives To describe current practices about the development of rapid guidelines and to provide advice about adequate methodology.

Methods We performed a systematic review, including grey literature, to identify (1) rapid guidelines, defined as guidelines produced in a shortened time frame, and (2) methodological manuals addressing its development.

Results We only documents by WHO and NICE that described methods and actual guidelines. The WHO handbook describes "rapid advice guidelines"; guidelines produced in response to a public health emergency in which WHO is required to provide rapid global leadership and guidance. This advice should be produced within 1 to 3 months and be evidence-informed, however, it may not be supported by full reviews of the evidence. We identified six WHO rapid guidelines and one methodological guidance paper based on a WHO guideline. NICE produces "short clinical guidelines"; guidelines that address only part of a care pathway, allowing rapid (11-13-month) development of guidance on aspects of care for which the NHS requires urgent advice. We identified 18 NICE short clinical guidelines.

Discussion Literature is lacking about rapid guidelines and the intended role appears to differ. Despite its relevance, there are few rapid guidelines published and clarity about the terminology is needed.

Implications for Guideline Developers We will provide a framework for those developing rapid guidelines, including practical advice and clarification about the terminology used.

\section{USING RAPID REVIEWS TO INFLUENCE GUIDANCE DEVELOPMENT IN THE EMERGENCY DEPARTMENT SETTING}

J Ramachandran. Kaiser Permanente, Southern California Permanente Medical Group, Pasadena, USA

10:1136/bmjqs-2013-002293.64
Background Decision making within health care organisations often requires rapid response to emergent, controversial or highimpact issues affecting clinical and operational practices.

Context CT imaging without oral contrast for patients admitted into the Emergency Department (ED) for abdominal/pelvic pain has been proposed as a viable option to reduce the risk of contrast-induced nephropathy and allergic reactions, as well as emergency room delays and overall length of stay (LOS) in the ED. A centralised evidence assessment unit within a large health care organisation was asked to conduct a rapid evidence review to inform the development of evidence-based guidance.

Description of Best Practice A 5 -step rapid review process was initiated, including: 1) Communicating with key stakeholders to determine relevant populations, interventions, comparisons, outcomes, timing and settings (PICOTS); 2) Conducting a comprehensive evidence search using a pre-established list of key databases and other sources to identify high-quality guidelines, systematic reviews and clinical trials evaluating the efficacy and diagnostic accuracy of conducting abdominal CT with and without oral contrast agents; 3) selecting and abstracting data from relevant studies; 4) evaluating and synthesising the literature; and 5) translating results for clinical/operational decision making. Findings of low- to moderate-quality evidence across outcomes, combined with operational and resource data, resulted in a decision not to implement the practice.

Lessons for Guideline Developers, Adaptors, Implementers, and/or Users Coupled with expert input from knowledgeable clinicians and stakeholders, rapid evidence reviews can be critical to shaping evidence-based guidance in Emergency Department settings.

\section{DO MODELS OF RAPID GUIDELINE UPDATES FIT WITHIN THE CURRENT AUSTRALIAN GUIDELINE STANDARDS? AN EXAMPLE FROM THE NATIONAL STROKE FOUNDATION CLINICAL GUIDELINES}

K Hill, L Wright. National Stroke Foundation, Melbourne, Australia

\section{0:1136/bmjas-2013-002293.65}

Background Clinical guideline recommendations are developed to assist health professionals to make evidence-based decisions. This is reliant on having the most up-to-date evidence available. The current Australian National Health and Medical Research Council (NHMRC) standards require guidelines to be updated within five years. An online process which provides transparency and enables timely changes/updates, such as the wiki platform developed and used by the Cancer Council of Australia (CCA), would provide a more useful platform to update guidelines but is currently not considered within the NHMRC standards.

Objectives To evaluate if using the CCA wiki platform to update stroke guidelines would meet NHMRC standards.

Methods We reviewed a potential CCA wiki platform ("wiki") model against the existing NHMRC standards (2011) to determine compatibility and identify where changes to the wiki model might be required.

Results The processes utilising the wiki were methodologically robust and were deemed to comply with $45 / 50$ of the mandatory elements of the NHMRC standards with minor changes needed to comply with the other five elements. Difficulties arise 